\title{
Detection and Measurement of the Abrupt Change of the Power Parameters of the Fast-Fluctuating Gaussian Random Process
}

\author{
Oleg V Chernoyarov ${ }^{1,2, *}$, Mahdi M Shahmoradian ${ }^{1}$, Alexandra V Salnikova ${ }^{2,3}$ and Ilya A Buravlev ${ }^{3}$ \\ ${ }^{1}$ National Research University "Moscow Power Engineering Institute”, 14, Krasnokazarmennaya st., Moscow, 111250 , Russia \\ ${ }^{2}$ International Laboratory of Statistics of Stochastic Processes and Quantitative Finance of National Research Tomsk State \\ University, 36, Lenin Avenue, Tomsk, 634050, Russia \\ ${ }^{3}$ Voronezh State Technical University, 14, Moscow Avenue, Voronezh, 394026, Russia \\ ${ }^{*}$ Corresponding author
}

\begin{abstract}
We introduce the technically simple approach to determining the abrupt change of the unknown mathematical expectation and dispersion of the low-frequency fast-fluctuating Gaussian random process against white noise. For this purpose, we determine new approximations of the decision statistics for various hypotheses, we carry out their maximization in terms of unknown parameters, and we develop the block diagrams for the corresponding detectors and measurers in the form of the comparatively simple single-channel units. For the analytical analysis of the performance of the synthesized algorithms, the asymptotically exact expressions for their characteristics, specifically - type I and type II error probabilities (when an abrupt change point is detected) and conditional biases and variances of the estimates (when measuring the parameters of the analyzed random process), are obtained by means of local Markov approximation method. The experimental testing of the presented theoretical results is implemented by the methods of statistical computer simulation.
\end{abstract}

Keywords-random process; abrupt change; detection; estimation; maximum likelihood method; parametrical prior uncertainty; local Markov approximation method; statistical computer simulation

\section{INTRODUCTION}

The problem of the statistical analysis of the abrupt change (i.e., instantaneous jumping at the some time) of the mathematical expectation and dispersion of the random process is of a great importance in the fields of technical and medical diagnostics, the control theory, in data processing, etc. [1-3, etc.]. In certain studies, the statement of this problem is accompanied by the assumption that the observable data realization has a normal distribution. As a rule, the additional restrictions are also imposed. Thus, in [1] it is presupposed that the processed samples are statistically independent, while [2,3] are concerned with the autoregressive models of the information process mainly, etc. Besides, in many cases the synthesis of detection and estimation algorithms for the abrupt change is usually conducted in the conditions of complete prior certainty regarding spurious parameters of the analyzed random process. In the relative few papers dealing with the statistical analysis of the abrupt change of Gaussian processes with unknown parameters, there are described comparatively complex iterative algorithms only operable in case of the very high signal-to-noise ratios (SNRs) [1, etc.].

In the present study, we consider the problem of the analysis of the abrupt change in the power parameters of the random process, presupposing that its fluctuations are fast (strong condition) and that its spectral density is approximately uniform within the specified bandwidth (weak condition). In terms of the technique introduced in [4], we suggest a technically simple approach to determining the moment of the stepwise change in the unknown mathematical expectation and dispersion of the band Gaussian random process and to their measurement before and after jumping. And we illustrate how to effectively overcome the parametrical prior uncertainty under arbitrary SNRs.

\section{ABRUPt CHANGE OF THE DiSPERSION OF THE RANDOM PROCESS}

At first, we consider the problem of the abrupt change of the unknown dispersion of the random process at the unknown point in time. We presuppose that other parameters of the random process are a priori known. And we write down such process analytically as follows

$$
\xi(t)=a+\left[\sigma_{1}+\left(\sigma_{2}-\sigma_{1}\right) \theta\left(t-\lambda_{0}\right)\right] v(t) .
$$

The designations are: $\theta(t)=0$, if $t<0$, and $\theta(t)=1$, if $t \geq 0$ - Heaviside function, $\lambda_{0}-$ the moment of possible stepwise change, $a$ - mathematical expectation of the process $\xi(t), \sigma_{1}, \sigma_{2}-$ mean square deviations of the process $\xi(t)$ under $t<\lambda_{0}$ and $t \geq \lambda_{0}$, accordingly, and $v(t)$ - stationary centered Gaussian random process possessing spectral density

$$
G(\omega)=\frac{2 \pi}{\Omega}\left\{\begin{array}{l}
1,|\omega| \leq \Omega / 2, \\
0,|\omega|>\Omega / 2
\end{array}\right.
$$

Here $\Omega$ is the bandwidth of the process $v(t)$. 
We believe that the process (1) is observed against additive Gaussian white noise $n(t)$ with one-sided spectral density $N_{0}$. As a result, the mix

$$
x(t)=\xi(t)+n(t), \quad t \in[0, T]
$$

Can now be observed. The fluctuations of the process $\xi(t)$ are now considered as "fast", so the following condition is satisfied

$$
\mu_{\min }=T_{\min } \Omega / 4 \pi>>1,
$$

where $T_{\min }=\min \left(\lambda_{0}, T-\lambda_{0}\right)$. With the observable realization (3), it is necessary to detect the abrupt change point and to estimate the parameters $\lambda_{0} \in\left[\Lambda_{1}, \Lambda_{2}\right], \sigma_{1}, \sigma_{2}$.

For the synthesis of the detection algorithm of the process $\xi(t)$ dispersion stepwise change, we separate the two possible cases (two hypotheses): 1) $\sigma_{1}=\sigma_{2}$, i.e. jumping is absent $\left(H_{0}\right.$ hypothesis); 2) $\sigma_{1} \neq \sigma_{2}$ ( $H_{1}$ hypothesis). The problem of the specified hypotheses testing is solved by means of the maximum likelihood method. For this purpose, with the results of the previous studies [4-6] in mind, we write down the expressions for the decision statistics (logarithms of the functionals of likelihood ratio) for hypotheses $H_{0}, H_{1}$ against alternative $H: x(t)=n(t)$ as

$$
\begin{gathered}
H_{0}: L_{0}\left(d_{1}\right)=\frac{d_{1}}{N_{0}\left(N_{0}+d_{1}\right)} \int_{0}^{T} y^{2}(t) d t+\frac{2 a}{N_{0}+d_{1}} \int_{0}^{T} x(t) d t- \\
-\frac{a^{2} T}{N_{0}+d_{1}}-\frac{\Omega T}{4 \pi} \ln \left(1+\frac{d_{1}}{N_{0}}\right), \\
L_{1}\left(\lambda, d_{1}, d_{2}\right)=\frac{d_{1}}{N_{0}\left(N_{0}+d_{1}\right)} \int_{0}^{\lambda} y^{2}(t) d t+\frac{2 a}{N_{0}+d_{1}} \int_{0}^{\lambda} x(t) d t+ \\
+\frac{d_{2}}{N_{0}\left(N_{0}+d_{2}\right)} \int_{\lambda}^{T} y^{2}(t) d t+\frac{2 a}{N_{0}+d_{2}} \int_{\lambda}^{T} x(t) d t-\frac{a^{2} \lambda}{N_{0}+d_{1}}- \\
-\frac{a^{2}(T-\lambda)}{N_{0}+d_{2}}-\frac{\Omega \lambda}{4 \pi} \ln \left(1+\frac{d_{1}}{N_{0}}\right)-\frac{\Omega(T-\lambda)}{4 \pi} \ln \left(1+\frac{d_{2}}{N_{0}}\right) .
\end{gathered}
$$

Here $y(t)=\int_{-\infty}^{\infty} x\left(t^{\prime}\right) h\left(t-t^{\prime}\right) d t^{\prime}$ is the output signal of the filter with the transfer function $H(\omega)$, satisfying the condition $|H(\omega)|^{2}=\Omega G(\omega) / 2 \pi(2)$, and $\lambda, d_{1}, d_{2}$ are current values of the parameters $\lambda_{0}, d_{01}=2 \pi \sigma_{1}^{2} / \Omega, d_{02}=2 \pi \sigma_{2}^{2} / \Omega$, accordingly. The choice is made in favor of the presence of the abrupt change, if $[4,6,7]$

$$
\max _{\lambda \in\left[\Lambda_{1}, \Lambda_{2}\right], d_{1}, d_{2}} L_{1}\left(\lambda, d_{1}, d_{2}\right)-\max _{d_{1}} L_{0}\left(d_{1}\right)>c,
$$

where $c$ is the threshold, calculated according to the specified optimality criterion.

The maximization of the functionals (5) with respect to variables $d_{1}, d_{2}$ can be performed analytically. As a result, the maximum likelihood algorithm (6) for detection of the abrupt change in the unknown dispersion of the low-frequency Gaussian random process takes the form of

$$
\max _{\lambda \in\left[\Lambda_{1}, \Lambda_{2}\right]} M(\lambda)_{\substack{H_{0} \\ H_{0}}}^{H_{1}} C
$$

$$
M(\lambda)=\ln \left[M_{3} / M_{2}(\lambda)\right]-(\lambda / T) \ln \left[M_{1}(\lambda) / M_{2}(\lambda)\right]
$$

and it is an invariant to the spectral density of the white noise. In Eq. (7) it is designated as:

$$
\begin{gathered}
M_{1}(\lambda)=\frac{1}{\lambda}\left[\int_{0}^{\lambda} y^{2}(t) d t-2 a \int_{0}^{\lambda} x(t) d t+a^{2} \lambda\right], \\
M_{2}(\lambda)=\frac{1}{T-\lambda}\left[\int_{\lambda}^{T} y^{2}(t) d t-2 a \int_{\lambda}^{T} x(t) d t+a^{2}(T-\lambda)\right], \\
M_{3}=\frac{1}{T}\left[\int_{0}^{T} y^{2}(t) d t-2 a \int_{0}^{T} x(t) d t+a^{2} T\right] .
\end{gathered}
$$

The decision detection rule (7) can be reduced to a simpler form processing the centered realization $\widetilde{x}(t)=x(t)-a$ instead of the initial realization of the observable data $x(t)(1)$. Then, we can write down the functionals $M_{1}(\lambda), M_{2}(\lambda), M_{3}$ in Eq. (7) as follows

$$
M_{1}(\lambda)=\frac{1}{\lambda} \int_{0}^{\lambda} \tilde{y}^{2}(t) d t, \quad M_{2}(\lambda)=\frac{1}{T-\lambda} \int_{\lambda}^{T} \tilde{y}^{2}(t) d t
$$

$$
H_{1}: M_{3}=\frac{1}{T} \int_{0}^{T} \tilde{y}^{2}(t) d t
$$

where $\widetilde{y}(t)=\int_{-\infty}^{\infty} \widetilde{x}\left(t^{\prime}\right) h\left(t-t^{\prime}\right) d t^{\prime}$, and $h(t)$ is determined in the same way as in Eq. (5).

The expressions (7), (8) define the structure of the maximum likelihood detector of the abrupt change in the 
unknown dispersion of the Gaussian random process. One of its possible implementation is shown in the form of block diagram selected by dashed line in Figure 1. Here the designations are as follows: 1 is the switch that is open for time $[0, T] ; 2$ is the substractor; 3 is a filter with transfer function $H(\omega) / \sqrt{T} ; 4$ is the squarer; 5 is an integrator; 6 is a delay line for the period $T$; 7 is the ramp generator $\lambda / T ; 8$ is the divider; 9 is the logarithmic amplifier; 10 is the multiplier; 11 is the peak detector; 12 is the threshold device, which is carrying out comparison of an output signal of the peak detector with a threshold $c$ within the interval $\left[\Lambda_{1}, \Lambda_{2}\right]$ and deciding upon the presence of the abrupt change in the dispersion of random process, if the threshold is exceeded, or deciding upon the absence of such abrupt change, if the threshold is not exceeded.

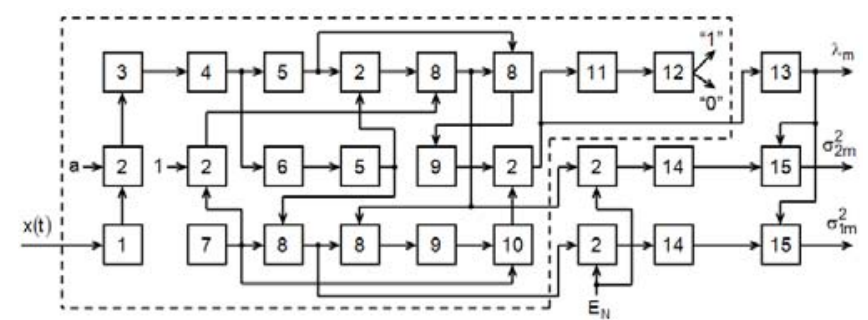

FIGURE I. BLOCK DIAGRAM OF THE DETECTOR/MEASURER OF THE ABRUPT CHANGE IN THE DISPERSION OF THE GAUSSIAN RANDOM PROCESS.

Let us suppose now that abrupt change in the dispersion of the random process $\xi(t)$ is realized with probability 1 within the interval $\left[\Lambda_{1}, \Lambda_{2}\right]$. And it is necessary to measure the change-point time $\lambda_{0}$ jointly with parameters $\sigma_{1}, \sigma_{2}$. Using Eq. (5), for the maximum likelihood estimates $\lambda_{m}, \sigma_{1 m}, \sigma_{2 m}$ of the unknown values $\lambda_{0}, \sigma_{1}, \sigma_{2}$ we obtain

$$
\begin{gathered}
\lambda_{m}=\underset{\lambda \in\left[\Lambda_{1}, \Lambda_{2}\right]}{\arg \max }\left\{\ln \left[\frac{M_{3}}{M_{2}(\lambda)}\right]-\frac{\lambda}{T} \ln \left[\frac{M_{1}(\lambda)}{M_{2}(\lambda)}\right]\right\}, \\
\sigma_{1 m}^{2}=M_{1}\left(\lambda_{m}\right)-E_{N}, \quad \sigma_{2 m}^{2}=M_{2}\left(\lambda_{m}\right)-E_{N} .
\end{gathered}
$$

where $E_{N}=N_{0} \Omega / 4 \pi$ is mean power of the noise $n(t)$ within bandwidth of the process $\xi(t)$, and $M_{1}(\lambda), M_{2}(\lambda), M_{3}$ are determined according to Eq. (8).

The measurer (10) can be implemented in the form of the block diagram shown in Figure 1, from which the peak detector 11 and the threshold device 12 should be excluded. Other designations are the following: 13 is the retriever of the location of the input signal greatest maximum (extremator) within the interval $\left[\Lambda_{1}, \Lambda_{2}\right] ; 14$ is the nonlinear element with characteristic $f(x)=\max (0, x) ; 15$ is the sampling device forming to its output the input signal sample at the instant time determined by the value $\lambda_{m}$ as output signal.
In order to determine the efficiency of the synthesized detection (7) and estimation (9) algorithms, we found the asymptotically exact (with increasing $\mu_{\min }(4)$ ) expressions for their characteristics such as type I and II error probabilities (for algorithm (7)) and conditional biases and variances of estimates (for algorithm (9)), using a local Markov approximation method $[8,9]$. We also carried out experimental tests for the performance of the detector (7) and the measurer (9) by methods of statistical computer simulation. As a result, we established that the detector (7) and the measurer (9) are operable, and theoretical formulas for type I and II error probabilities and conditional biases and variances of the decided estimates well conform to corresponding experimental data in a wide range of parameter values of the process $\xi(t)$. And we showed that processing algorithms (7), (9) can be used in the analysis of many widespread non-Gaussian random processes without appreciable loss in accuracy.

\section{ABRUPT CHANGE IN THE MATHEMATICAL}

\section{EXPECTATION AND THE DISPERSION OF THE RANDOM PROCESS}

We now consider a case, when both the dispersion and the mathematical expectation of the process $\xi(t)$ are unknown, and they can abruptly change at the time $\lambda_{0}$, so

$$
\xi(t)=a_{01}+\left(a_{02}-a_{01}\right) \theta\left(t-\lambda_{0}\right)+\left[\sigma_{1}+\left(\sigma_{2}-\sigma_{1}\right) \theta\left(t-\lambda_{0}\right)\right] v(t) .
$$

Here $a_{01}, a_{02}$ are mathematical expectations of the process $\xi(t)$ under $t<\lambda_{0}$ and $t \geq \lambda_{0}$, accordingly, and other designations coincide with the ones used in Eq. (1). Subject to Eq. (4), with realization (3), (10) it is necessary to detect the change-point time and to estimate the parameters $\lambda_{0} \in\left[\Lambda_{1}, \Lambda_{2}\right], a_{01}, a_{02}, \sigma_{1}, \sigma_{2}$.

As in section 2, while synthesizing the detection algorithm of the abrupt change in power parameters of the process $\xi(t)$ we separate the two possible cases: 1) $a_{01}=a_{02}, \sigma_{1}=\sigma_{2}$, i.e. abrupt change is absent ( $H_{0}$ hypothesis); 2) $a_{01} \neq a_{02}$ and/or $\sigma_{1} \neq \sigma_{2}$ ( $H_{1}$ hypothesis). We will solve the problem of the specified hypothesis testing by means of the maximum likelihood method and, following [5, 6], we will write down expressions for the logarithms of the functionals of likelihood ratio under hypotheses $H_{0}$ and $H_{1}$ against alternative $H$ : $x(t)=n(t)$ in the form of 


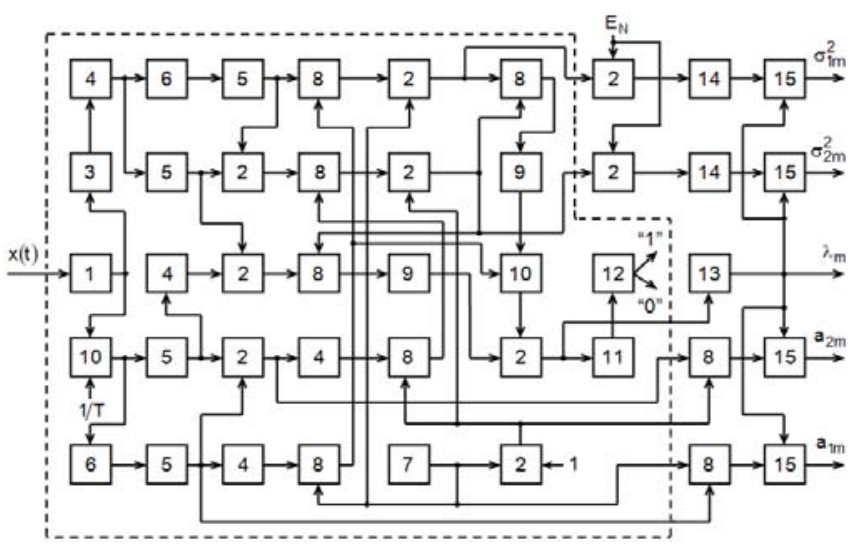

FIGURE II. BLOCK DIAGRAM OF THE DETECTOR/MEASURER OF THE ABRUPT CHANGE IN THE MATHEMATICAL EXPECTATION AND THE DISPERSION OF THE GAUSSIAN RANDOM PROCESS.

$$
\begin{gathered}
H_{0}: \begin{array}{c}
L_{0}\left(a_{1}, d_{1}\right)=\frac{d_{1}}{N_{0}\left(N_{0}+d_{1}\right)} \int_{0}^{T} y^{2}(t) d t+\frac{2 a_{1}}{N_{0}+d_{1}} \int_{0}^{T} x(t) d t- \\
\frac{a_{1}^{2} T}{N_{0}+d_{1}}-\frac{\Omega T}{4 \pi} \ln \left(1+\frac{d_{1}}{N_{0}}\right) \\
L_{1}\left(\lambda, a_{1}, a_{2}, d_{1}, d_{2}\right)=\frac{d_{1}}{N_{0}\left(N_{0}+d_{1}\right)} \int_{0}^{\lambda} y^{2}(t) d t+ \\
H_{1}:+\frac{2 a_{1}}{N_{0}+d_{1}} \int_{0}^{\lambda} x(t) d t+\frac{d_{2}}{N_{0}\left(N_{0}+d_{2}\right)} \int_{\lambda}^{T} y^{2}(t) d t+ \\
+\frac{2 a_{2}}{N_{0}+d_{2}} \int_{\lambda}^{T} x(t) d t-\frac{a_{1}^{2} \lambda}{N_{0}+d_{1}}-\frac{a_{2}^{2}(T-\lambda)}{N_{0}+d_{2}}- \\
-\frac{\Omega \lambda}{4 \pi} \ln \left(1+\frac{d_{1}}{N_{0}}\right)-\frac{\Omega(T-\lambda)}{4 \pi} \ln \left(1+\frac{d_{2}}{N_{0}}\right)
\end{array}
\end{gathered}
$$

Here $y(t), \lambda, d_{1}, d_{2}$ are determined in the same way as in Eq. (5), and $a_{1}, a_{2}$ are current values of the parameters $a_{01}$, $a_{02}$, accordingly. Similarly to Eq. (6), the decision upon the presence of the abrupt change is made, if the following inequality is satisfied

$$
\max _{\lambda \in\left[\Lambda_{1}, \Lambda_{2}\right], a_{1}, a_{2} d_{1}, d_{2}} L_{1}\left(\lambda, a_{1}, a_{2}, d_{1}, d_{2}\right)-\max _{a_{1}, d_{1}} L_{0}\left(a_{1}, d_{1}\right)>c,
$$

where $c$ is the threshold, calculated according to the specified optimality criterion.

Maximization with respect to variables $a_{1}, a_{2}, d_{1}, d_{2}$ in Eq. (12) can be performed analytically. As a result, the maximum likelihood detection algorithm of the abrupt change in unknown mathematical expectation and/or dispersion of the Gaussian random process takes the form of

$$
\begin{gathered}
\max _{\lambda \in\left[\Lambda_{1}, \Lambda_{2}\right]} M(\lambda){\underset{<}{H_{0}}}_{>}^{H_{1}} c, \\
M(\lambda)=\ln \left[M_{3} / M_{2}(\lambda)\right]-(\lambda / T) \ln \left[M_{1}(\lambda) / M_{2}(\lambda)\right]
\end{gathered}
$$

and is invariant to the spectral density of the white noise. Here it is designated:

$$
\begin{gathered}
M_{1}(\lambda)=\frac{1}{\lambda}\left[\int_{0}^{\lambda} y^{2}(t) d t-\frac{1}{\lambda}\left(\int_{0}^{\lambda} x(t) d t\right)^{2}\right], \\
M_{2}(\lambda)=\frac{1}{T-\lambda}\left[\int_{\lambda}^{T} y^{2}(t) d t-\frac{1}{T-\lambda}\left(\int_{\lambda}^{T} x(t) d t\right)^{2}\right], \\
M_{3}=\frac{1}{T}\left[\int_{0}^{T} y^{2}(t) d t-\frac{1}{T}\left(\int_{0}^{T} x(t) d t\right)^{2}\right] .
\end{gathered}
$$

The detector (13) can be implemented in the form of the block diagram selected in Figure 2 by dashed line. Here designations are the same as in Figure 1.

Let us suppose now that abrupt change in the mathematical expectation and/or the dispersion of the random process $\xi(t)$ is realized with probability 1 within the interval $\left[\Lambda_{1}, \Lambda_{2}\right]$. And it is necessary to measure the change-point time $\lambda_{0}$ jointly with the parameters $a_{01}, a_{02}, \sigma_{1}, \sigma_{2}$. Using Eq. (11), for the maximum likelihood estimates $\lambda_{m}, a_{1 m}, a_{2 m}, \sigma_{1 m}, \sigma_{2 m}$ of the unknown values $\lambda_{0}, a_{01}, a_{02}, \sigma_{1}, \sigma_{2}$ we obtain

$$
\lambda_{m}=\underset{\lambda \in\left[\Lambda_{1}, \Lambda_{2}\right]}{\arg \max }\left\{\ln \left[\frac{M_{3}}{M_{2}(\lambda)}\right]-\frac{\lambda}{T} \ln \left[\frac{M_{1}(\lambda)}{M_{2}(\lambda)}\right]\right\},
$$

$$
a_{1 m}=\frac{1}{\lambda_{m}} \int_{0}^{\lambda_{m}} x(t) d t, \quad a_{2 m}=\frac{1}{T-\lambda_{m}} \int_{\lambda_{m}}^{T} x(t) d t
$$

$$
\sigma_{1 m}^{2}=M_{1}\left(\lambda_{m}\right)-E_{N}, \quad \sigma_{2 m}^{2}=M_{2}\left(\lambda_{m}\right)-E_{N} .
$$

where $E_{N}$ and $M_{1}(\lambda), M_{2}(\lambda), M_{3}$ are determined from Eqs. (9) and (14), correspondently.

The measurer (15) can be implemented in the form of the block diagram shown in Figure 2, from which the peak detector 
11 and the threshold device 12 should be excluded. Other designations coincide with the ones specified in Figure 1.

In order to determine the efficiency of the synthesized detection (13) and estimation (15) algorithms, we found the asymptotically exact (with increasing $\mu_{\min }$ (4)) expressions for their characteristics, such as type I and II error probabilities (for algorithm (13)) and conditional biases and variances of estimates (for algorithm (15)), using a local Markov approximation method [8,9]. We carried out experimental testing of the performance of the detector (13) and the measurer (15) by means of statistical computer simulation. As a result, we established that the detector (13) and the measurer (15) are operable, and theoretical formulas for type I and II error probabilities, as well as the conditional biases and variances of the decided estimates well conform to the corresponding experimental data in a wide range of parameter values of the process $\xi(t)$. Also, we showed that algorithms (13), (15) can be used in the analysis of many widespread non-Gaussian random processes without appreciable loss in accuracy.

\section{CONCLUSION}

In order to detect the abrupt change point in the fastfluctuating Gaussian process and to measure its jumping and constant parameters, the maximum likelihood method can be effectively applied. This approach allows us to obtain the algorithms for determining abrupt change in the unknown power parameters of the random process, while neglecting the values of the order of the correlation time of the analyzed random process. These algorithms are technically the simplest ones in comparison with the common analogues. Additional researches show that the detectors and the measurers synthesized by means of the introduced approach can also be used in the analysis of the abrupt change of the statistical characteristics of the non-Gaussian low-frequency random processes and bring no great losses in performance. The obtained results are proved by computer simulation.

\section{ACKNOWLEDGMENT}

The reported study was supported by the Ministry of Education and Science of the Russian Federation (research project No. 2.3208.2017/PC).

\section{REFERENCES}

[1] A.A. Zhiglyavsky, A.E. Kraskovsky, Detection of Abrupt Changes in Random Processes for Radio engineering Tasks (in Russian). Leningrad: Leningrad State University, 1988.

[2] N. Kligene, L. Tel'ksnis, "Methods to Determine the Times when the Properties of Random Processes Change", Automation and Remote Control, vol. 41, pp. 1241-1283, October 1983.

[3] M. Basseville, I.V. Nikiforov, Detection of Abrupt Changes: Theory and Application. New Jersey: Prentice-Hall, Englewood Cliffs, 1993.

[4] O.V. Chernoyarov, B.I. Shakhtarin, A.P. Ermakov, D.K. Proskurin, "The New Approach to the Detection of the Abrupt Change of Fast Fluctuating Random Processes in the Conditions of Parametric Prior Uncertainty", Proceedings of the International Conference on Automatic Control Theory and Application (ACTA 2014), vol. 2, pp. 9-13, Thailand, June 2014.

[5] O.V. Chernoyarov, M.M. Shahmoradian, K.S. Kalashnikov, "The Decision Statistics of the Gaussian Signal against Correlated Gaussian
Interferences", Proceedings of the 2016 International Conference on Mathematical, Computational and Statistical Sciences and Engineering (MCSEE2016), pp. 426-431, China, October 2016.

[6] O.V. Chernoyarov, Sai Si Thu Min, A.V. Salnikova, M. Kuba, "Detection and Estimation of Abrupt Changes in Gaussian Random Processes with Unknown Parameters", Proceedings of the 10th International Conference "ELEKTRO2014", pp. 46-51, Slovakia, May 2014.

[7] O.V. Chernoyarov, A.V. Salnikova, D.S. Rozhkova, "Abrupt Change of the Mathematical Expectation of the Random Process with Unknown Intensity", Applied Mathematical Sciences, vol. 9, pp. 6891-6908, November 2015.

[8] O.V. Chernoyarov, Sai Si Thu Min, A.V. Salnikova, B.I. Shakhtarin, A.A. Artemenko, "Application of the Local Markov Approximation Method for the Analysis of Information Processes Processing Algorithms with Unknown Discontinuous Parameters", Applied Mathematical Sciences, vol. 8, pp. 4469-4496, August 2014.

[9] O.V. Chernoyarov, A.V. Salnikova, A.E. Rozanov, M. Marcokova, "Statistical Characteristics of the Magnitude and Location of the Greatest Maximum of Markov Random Process with Piecewise Constant Drift and Diffusion Coefficients", Applied Mathematical Sciences, vol. 8 , pp. 7341-7357, October 2014. 